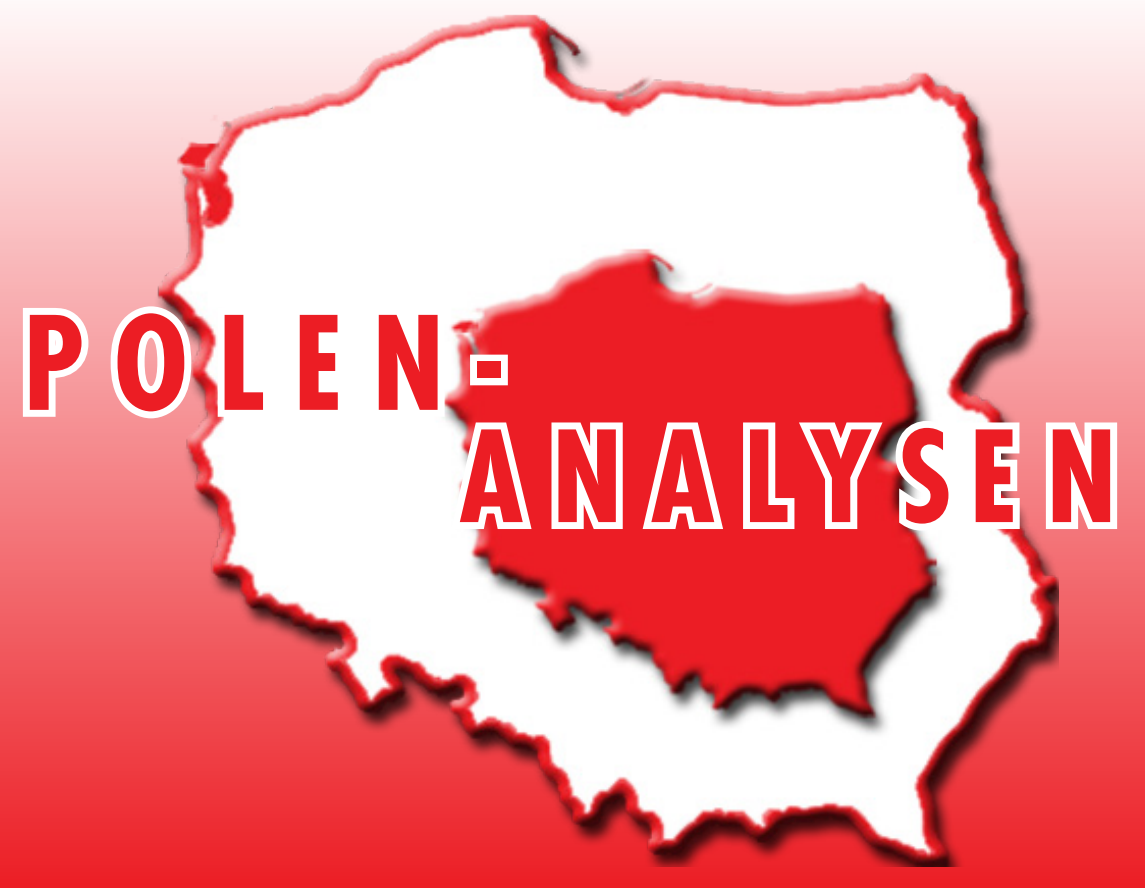

www.laender-analysen.de/polen

\title{
DER ENERGIESEKTOR
}

ANALYSE

Energie - Herausforderungen für Polen

Adam Grzeszak, Warschau

- GRAFIKEN ZUM TEXT

Der polnische Energiemix

- CHRONIK

Vom 1. bis zum 14. Mai 2012

- VERANSTALTUNG

Deutsch-Polnische Sommerakademie des Deutschen Polen-Instituts in Darmstadt 


\title{
Energie - Herausforderungen für Polen
}

\author{
Adam Grzeszak, Warschau
}

\section{Zusammenfassung}

Polen steht vor großen Herausforderungen im Bereich der Energiepolitik. Das derzeitige Modell, das noch aus der Zeit des kommunistischen Systems stammt, basiert auf der Kohle-Monokultur großer Kraftwerke, deren Durchschnittsalter über 30 Jahre beträgt, und auf einer - wenn auch abnehmend - energieintensiven Wirtschaft. In den nächsten Jahren muss Polen ein neues Modell für den Energiesektor entwickeln. Dies wird nicht nur eine große wirtschaftliche und juristische, sondern auch eine politische und gesellschaftliche Herausforderung. Viele polnische Politiker betrachten die Energiepolitik nur im Kontext der polnisch-russischen Beziehungen und träumen von Energieautarkie.

$\mathrm{O}$ bjektiv betrachtet ist Polen ein Land mit hoher Energiesicherheit - der Bedarf an Energie wird überwiegend aus heimischen Quellen gespeist. Subjektiv allerdings empfinden die Polen ihre Situation im Bereich Energie als sehr unsicher. Daher ist das Problem der Energiesicherheit seit Jahren einer der zentralen Punkte der öffentlichen Debatte und politischer Konflikte. Dabei wird mehr Gewicht auf "Sicherheit" als auf "Energie" gelegt. Unter Politikern besteht die ausgeprägte Tendenz, die Probleme des weit gefassten Energiesektors in den Kategorien der nationalen Sicherheit zu betrachten.

In die Geschichte ging die Aussage von Janusz Steinhoff, stellvertretender Ministerpräsident und Wirtschaftsminister (1997-2001), ein, der in der Diskussion über das damalige Regierungsprojekt des Baus einer Unterwassergaspipeline von Norwegen und Dänemark nach Polen sagte: »Sicherheit hat keinen Preis.« Das Projekt war damals von Experten als unrealistisch, kostspielig und ökonomisch sinnlos kritisiert worden. Die Politiker sahen jedoch einen politischen Sinn darin, nämlich die Möglichkeit, Polen vom russischen Gas unabhängig zu machen, und fanden, dass dabei der Preis keine Rolle spiele. Und obwohl die Investition nicht über das Stadium der Diskussion und erster Analysen hinausging, war sie viele Jahre lang Gegenstand hitzigen Streits. Noch heute taucht sie in den Diskussionen als vertane Chance auf. Unlängst erst bedauerte Schatzminister Mikołaj Budzanowski öffentlich, dass diese Gaspipeline nicht gebaut worden ist.

Energieprojekte, die auf politischen Konzepten basieren, gibt es viele. Ein Beispiel ist das Projekt, die ukrainische Ölpipeline Odessa-Brody nach Danzig (Gdańsk) zu verlängern. Sie war von der Ukraine mit dem Ziel gebaut worden, vom russischen Erdöl unabhängiger zu werden. In der Praxis wird sie allerdings von russischen Erdölgesellschaften genutzt, denn sie verbindet den Hafen von Odessa mit dem nördlichen Abschnitt der Ölpipeline Druschba, über die russisches Erdöl nach Europa exportiert wird.
Das Projekt sah die Verlängerung dieser Pipeline bis nach Polen vor, so dass polnische Raffinerien Erdöl erhalten könnten. Das grundlegende Problem ist dabei, eine ausreichende Menge Erdöl, das aus anderen als aus russischen Quellen stammt, nach Odessa zu liefern, um es nach Polen zu pumpen. Für dieses Projekt hatte sich der damalige Staatspräsident Lech Kaczyński stark engagiert, als die Partei seines Bruders Jarosław, Recht und Gerechtigkeit (Prawo i Sprawiedliwość - PiS), in der Regierungsverantwortung stand. Damals wurde versucht, eine Energiebrücke zwischen den zentralasiatischen Staaten und Danzig aufzubauen. Dabei wurde davon ausgegangen, dass das Erdöl aus Kasachstan und Aserbaidschan ans georgische Ufer des Schwarzen Meeres transportiert und von dort mit Tankern nach Odessa gebracht würde. Dann sollte es per Pipeline bis nach Płock gepumpt werden, wo sich die größte polnische Raffinerie (des Konzerns Orlen) befindet, sowie nach Danzig, wo die zweitgrößte Raffinerie Polens (des Konzerns Lotos) liegt und der Erdölhafen.

Dieses Projekt war nicht nur wirtschaftlich, sondern auch politisch allzu unrealistisch. Kasachstan war nicht daran interessiert, sich in einem so unsicheren Vorhaben zu engagieren, das einen deutlich antirussischen Charakter hatte, während Aserbaidschan nach der Inbetriebnahme der Ölpipeline Baku-Tiflis-Ceyhan (BTC) nicht über die ausreichende Menge an Erdöl verfügte. Obwohl das Projekt Odessa-Brody-Danzig starke politische Unterstützung sowohl von Polen als auch der Ukraine erfuhr (insbesondere in der Regierungszeit von Leonid Kutschma und Viktor Juschtschenko), wurde es nicht umgesetzt. Es wurde aber auch nicht vollständig aufgegeben. Die polnisch-ukrainisch-aserische Gesellschaft Sarmatia führt weiterhin Studien durch, die die Möglichkeiten des Baus einer solchen Pipeline prüfen.

\section{Die Abhängigkeit Polens von Russland}

In politischen Diskussionen wird Russland als die größte Gefahr für die Energiesicherheit Polens angesehen. Es 
herrscht die Überzeugung, dass der Kreml danach strebt, mit Hilfe der wirtschaftlichen Waffen wie Erdöl und Gas die Dominanz zu gewinnen. Tatsächlich ist Polen in hohem Maße von den russischen Lieferungen abhängig. Zirka 90 Prozent des von den polnischen Raffinerien verarbeiteten Erdöls und Gas zu zirka 70 Prozent kommen aus Russland. Diese Abhängigkeit ergibt sich aus historischen, geographischen und infrastrukturellen Gründen. Die wichtigsten Gas- und Erdölpipelines und Erdöllager entstanden in Zeiten des Kommunismus und hatten auch eine starke militärische Funktion - es ging um die Treibstoffherstellung hinter der Front in einem von sowjetischen Strategen geplanten Konflikt.

Auch nach 1989 war es Polen viele Jahre nicht gelungen, alternative Versorgungswege aufzubauen. Die einzige große Investition war der Bau der Transitgaspipeline Jamal, einer weiteren Verbindung Polens mit dem östlichen Lieferanten. Mit dieser Pipeline wird russisches Gas über Polen nach Deutschland geliefert und sie wurde zu einem weiteren Streitpunkt in den polnischrussischen Beziehungen. Der Eigentümer des polnischen Abschnitts ist die polnisch-russische Gesellschaft EuroPolGaz, die bisher auch ihr Betreiber war. Der internationale Vertrag, auf dessen Grundlage die Gaspipeline gebaut worden war, garantierte Russland zahlreiche Privilegien. Deren Interpretation führt zu nicht endenden Konflikten. Die Situation verschärfte sich im Laufe des letzten Jahres, als Polen die Richtlinie des Europäischen Parlaments und des Rates über gemeinsame Vorschriften für den Erdgasbinnenmarkt (2009/73/EG) implementierte. Erforderlich war daraufhin, die Bereiche Gaslieferung und Gashandel zu trennen. Betreiber der Pipeline wurde die staatliche Gesellschaft Gaz System (Betreiber des inländischen Gasleitungssystems); außerdem wurde die Regel des third party access eingeführt. Gazprom wehrt sich dagegen, indem es in Frage stellt, dass die Gaspipeline, die noch vor dem EU-Beitritt Polens gebaut worden war, EU-Recht unterliegt.

Dies ist bereits der zweite Konfliktpunkt neben dem Streit, der vor dem Schiedsgericht in Stockholm geführt wird und in dem es um die Preise für Gas geht, die die größte polnische Gasgesellschaft PGNiG zahlen muss. Unter den Abnehmern von Gazprom in der EU muss Polen zurzeit den höchsten Preis für russisches Gas zahlen (400-500 USD/1.000 $\mathrm{m}^{3}$ ) und will dies ändern. Der Preis wurde nach einer besonderen Formel berechnet, die den Gaspreis in Anlehnung an die Notierung des Ölpreises berechnet. Dies ist ein Punkt des langjährigen Vertrags (bis 2022), den Polen 1996 mit Russland über Gaslieferungen einging, als der Vertrag für den Bau der Jamal-Pipeline unterzeichnet wurde. Der Vertrag wurde seitdem viele Male neu verhandelt und geändert. Im Jahr 2006 nutzte Russland die polnischen Gas- probleme und legte eine neue Preisformel fest, die heute der Gegenstand des Streits ist.

\section{Das deutsch-russische Nord Stream-Projekt}

Bisher hat Polen nur in geringem Maße das Gas genutzt, das über die Jamal-Pipeline kommt. Der größte Teil der russischen Lieferungen kommt über die Gaspipelines der Ukraine und Belarus'. Dies bedeutet eine reale Gefahr für die Liefergarantien, denn die Konflikte von Gazprom mit der Ukraine und Belarus führten schon wiederholt dazu, dass die Gaslieferungen nach Polen ausgesetzt oder reduziert wurden. Solche Ereignisse verstärken in Polen Ängste und die Überzeugung, dass Russland die "Gaswaffe« einsetzt.

Ein anderer Fall, den Polen als Bestätigung für die feindlichen Absichten Russlands auffasste, war die Einstellung der Erdöllieferungen an die litauische Raffinerie in Mozejki (Litauen) genau in dem Moment, als der Betrieb von dem polnischen Ölkonzern Orlen gekauft worden war. Auch russische Ölkonzerne hatten sich um den Erwerb bemüht und als sie aus dem Wettbewerb ausgeschieden waren, teilte der russische Betreiber des Ölpipeline-Systems, Transneft, mit, dass der Abzweig der Druschba-Pipeline, der nach Litauen abgeht, defekt sei und kein Öl mehr geliefert werden könne. Seit dem Jahr 2006 wird Mozejki über den Seeweg versorgt, was die Rentabilität der Produktion verschlechtert und Orlen Verluste bringt. Den Russen ist es bisher nicht gelungen, die Pipeline zu reparieren.

Auch das Projekt der Nord Stream-Gaspipeline, die auf dem Boden der Ostsee verlegt wurde, wurde als Schlag für Polen interpretiert. Polen widersetzte sich der russisch-deutschen Investition, und Radosław Sikorski, zurzeit Außenminister und damals Verteidigungsminister in der Regierung von PiS, nannte die Vereinbarung über Nord Stream öffentlich einen »neuen Hitler-Stalin-Pakt«. In Polen herrscht die Überzeugung, dass das Hauptziel dieser Gaspipeline ist, den Transit über die Länder Osteuropas einzustellen, um leichter Druck auf die Staaten dieser Region ausüben zu können, ohne dadurch die großen Abnehmer in Westeuropa zu beeinträchtigen. Deutschland wird als Verbündeter Russlands und seiner Gaspolitik gesehen; die Russen gewinnen immer mehr Einfluss im deutschen Energiesektor, offenen und verdeckten, - so die Überzeugung. Als Symbol dafür gilt die Aufnahme des ehemaligen Bundeskanzlers Gerhard Schröder in den Aufsichtsrat des Nord Stream-Konsortiums. Die Vorhaben, Verbindungspipelines an der polnisch-deutschen Grenze zu bauen, wurden deshalb von einflussreichen Politikern des polnischen rechtskonservativen Lagers bekämpft. Polen würde das russische Gas via Deutschland erhalten und somit die bestehende Abhängigkeit verdoppelt. 
Ein besonderer Streitpunkt ist der Verlauf der Nord Stream-Pipeline im Gebiet des Hafens von Swinemünde (Świnoujście), wo der Seeterminal für Flüssiggas (LNG) gebaut wird. Diese Standardinvestition wird als Durchbruch bei der Diversifizierung von Gaslieferungen nach Polen behandelt. Im Jahr 2014 soll der Terminal in Betrieb genommen werden. Die Fahrrinne für die Schiffe mit Gaslieferungen kreuzt die Nord StreamPipeline. Der Streit betrifft die zu flache Verlegung der Gaspipeline, was die Möglichkeiten beschneiden kann, dass Schiffe mit großem Tiefgang an den polnischen Flüssiggasterminal liefern.

Der Bau des LNG-Terminals ist eine von mehreren Investitionen, die Alternativen für die polnische Gasversorgung schaffen sollen. Dazu gehört auch der Ausbau eines Hochdruck-Gaspipelinenetzes, die unlängst erfolgte Inbetriebnahme eines Gas-Interkonnektors in Teschen (Cieszyn) an der polnisch-tschechischen Grenze (mit einer Kapazität von 0,5 Mrd. $\mathrm{m}^{3}$ jährlich) sowie die Modernisierung der Installationen an der polnisch-deutschen Grenze bei Zgorzelec/Görlitz, die nun 1,5 Mrd. $\mathrm{m}^{3}$ jährlich pumpen können. Ähnliches ist auch an den Grenzen mit Litauen und der Slowakei geplant. Da es bei diesen Investitionen um die Integration Polens in das Gassystem der EU geht, wird ein Teil der Investitionen aus EU-Fonds mitfinanziert. Darüber hinaus werden zurzeit die unterirdischen Gasspeicher ausgebaut.

\section{Der Traum vom Schiefergas}

Die größten Hoffnungen weckt in Polen heute allerdings das Schiefergas. Polen hat eine der größten Schieferablagerungen in Europa und hofft, dass darin Erdgas und Erdöl zu finden sind. Diese Hoffnung haben die Amerikaner den Polen eingeimpft, die als erste die Fördertechnologie für sogenannte unkonventionelle Gasvorkommen beherrschten und für Investitionen in die weltweite Suche und Förderung werben. Im Jahr 2009 schätzte die Firma Wood Mackenzie die polnischen Schiefergasvorkommen auf $1.400 \mathrm{Mrd} . \mathrm{m}^{3}$ und das Advanced Research Institute auf $3.000 \mathrm{Mrd} . \mathrm{m}^{3}$. Die größte mediale Karriere machte jedoch die Information im Weltreport der U.S. Energie Information Agency, die von über 5.300 Mrd. $\mathrm{m}^{3}$ sprach. Sollten die Vorkommen tatsächlich so groß sein, würden sie den Bedarf Polens für mehrere hundert Jahre decken. Die Emotionen wurden unlängst vom Bericht des Staatlichen Geologischen Instituts in Warschau ein wenig abgekühlt, der in Zusammenarbeit mit dem U.S. Geological Survey erstellt wurde und die polnischen Vorkommen um ein vielfaches geringer schätzt. Demnach können sie maximal 1.920 Mrd. $\mathrm{m}^{3}$ Gas enthalten, sehr wahrscheinlich wird sich aber die förderbare Menge zwischen 346 und $768 \mathrm{Mrd} . \mathrm{m}^{3}$ bewegen. Diese Schätzungen basieren auf Daten über die Förde- rung ähnlicher Vorkommen in den USA. Der Bericht bewertete nicht die wirtschaftliche Seite, die aber eine Schlüsselbedeutung für die Gasförderung haben wird. Die Gasvorkommen liegen in Polen sehr viel tiefer als in den USA, durchschnittlich in einer Tiefe von 3.000 Metern. Das wird die Kosten für Bohrungen und Förderung deutlich erhöhen.

Die schnelle Suche und Förderung von Schiefergas wurde zur Priorität der Regierung von Ministerpräsident Donald Tusk, denn damit werden viele Hoffnungen verbunden. Im »schlechtesten " Fall wird davon ausgegangen, dass Polen im Bereich Gas autark wird, es fehlt aber auch nicht an Stimmen (insbesondere unter Politikern), dass das Gas eine "Goldader" sein könnte, die den nächsten Generationen Wohlstand garantieren wird. Häufig wird der Begriff »zweites Norwegen« verwendet, und es werden auch Vorschläge gemacht, einen Fonds für zukünftige Generationen nach norwegischem Vorbild zu gründen, in den der Gewinn aus der Gasförderung fließen soll. Zurzeit steht die Suche allerdings noch am Anfang. 18 Firmen haben eine Konzession für die Suche nach Gas aus unkonventionellen Vorkommen erhalten. Die meisten hat PGNiG, die größte polnische Gasgesellschaft, die vom Staatsschatz kontrolliert wird. Außerdem suchen viele große internationale Konzerne in Polen nach Gas, u. a. Chevron, ExxonMobile, Marathon und Eni. Bisher wurden einige Dutzend Tiefbohrungen ausgeführt und einige horizontale, bei denen ein hydraulic fracturing (das sogenannte Fracking) durchgeführt wurde. Aus inoffiziellen Informationen geht hervor, dass die Ergebnisse einiger Bohrungen, vor allem in Pommern, vielversprechend seien. Die Regierung von Ministerpräsident Tusk möchte, dass noch vor Beendigung der Wahlperiode (2015) die industrielle Gasförderung beginnt. Experten bezweifeln aber, dass dies realistisch sei. Allerdings verlangt Schatzminister Mikołaj Budzanowski von allen Energiegesellschaften, an denen der Staat ein Kontrollpaket besitzt, dass sie sich an der Gassuche oder zumindest an der Mitfinanzierung der Arbeiten beteiligen.

\section{Umweltschutz ist zweitrangig}

In Polen befassen sich zwei Ministerien gleichzeitig mit Energiefragen, was zu Spannungen und Konflikten führt. Formal zuständig ist der Wirtschaftsminister. Er vertritt Polen auf Energiekonferenzen der EU. Das Wirtschaftsministerium bereitet Energiegesetzesentwürfe und andere Dokumente, die die Energiepolitik des Staates betreffen, vor. Wirtschaftsminister ist Waldemar Pawlak, Vorsitzender der Polnischen Bauernpartei (Polskie Stronnictwo Ludowe-PSL), die gemeinsam mit der Bürgerplattform (Platforma Obywatelska - PO) die Regierung bildet. Das Verhältnis zwischen Ministerpräsident 
Tusk und Pawlak ist gegenwärtig schwierig, daher ist derjenige, der den größeren tatsächlichen Einfluss auf Energiefragen hat, der Schatzminister, der die Gesellschaften des Staatsschatzes beaufsichtigt.

Formal gesehen führt das Schatzministerium Privatisierungen staatlicher Firmen durch. Im Energiesektor gilt das Dogma, die Privatisierungen auf ein unentbehrliches Minimum zu beschränken. Die Unternehmen aus dem Bereich Transportinfrastruktur von Strom, Gas und Erdöl sind Eigentum des Staates. In anderen Gesellschaften (von denen die Mehrheit öffentliche Gesellschaften sind, die an der Börse notiert sind) beabsichtig der Staat sein Kontrollpaket zu halten, denn staatliches Eigentum gilt als die beste Garantie für Energiesicherheit.

Schatzminister Mikołaj Budzanowski ist ein Beamter ohne politische Ambitionen, daher zieht es der Ministerpräsident vor, mit seiner Vermittlung die Energiepolitik zu betreiben. Eine ähnliche Situation besteht mit Landwirtschaftsminister Marcin Korolec, der Polen in Klimaverhandlungen vertritt und gleichzeitig für die Vergabe von Konzessionen für die Suche und Förderung von Schiefergas verantwortlich ist. Er hat also eine strategische Aufgabe hinsichtlich der Suche und Erschließung der Vorkommen, wobei gleichzeitig die Weisung besteht, die Hindernisse aus Umweltschutzgründen nicht $\mathrm{zu}$ vermehren.

Fragen der Ökologie und Sicherheit bei der Förderung von Schiefergas werden als zweitrangig gegenüber dem strategischen Ziel der Energieunabhängigkeit angesehen. Obgleich die Methode des hydraulic fracturing bei der Förderung unkonventionellen Gases in Europa große Kontroversen hervorruft, sind sich in Polen alle politischen Kräfte einig, dass die Förderung so schnell wie möglich beginnen sollte, sogar wenn dies mit Kosten für die Umwelt verbunden sein sollte.

Das Thema Gas ist außerdem einer der wenigen Bereiche, in dem eine gewisse Kooperation zwischen der Regierungskoalition und der Opposition besteht. Die $P O$-Regierung setzt einen Teil der Projekte fort, die die Regierung von PiS begonnen hat. Einige hohe Beamte aus dem Bereich Energie der Vorgängerregierung haben auch in der jetzigen Regierung einen Posten erhalten. An sich gibt es außer der kleinen außerparlamentarischen Opposition Grüne 2004 (Zieloni 2004) keine Kräfte von Bedeutung, die dagegen kämpfen, Schiefergas zu gewinnen. Die Grünen 2004 werden von José Bové unterstützt, einem französischen Globalisierungsgegner, Vizevorsitzender des Ausschusses für Landwirtschaft und ländliche Entwicklung des Europäischen Parlaments. Dies stößt allerdings eine Verschwörungstheorie an - in Polen herrscht die Überzeugung, dass hinter allen Aktivitäten, die die Förderung von Schiefergas in Europa ver- hindern sollen, Gazprom steht. Sogar Greenpeace Polen engagiert sich nicht in Sachen Schiefergas, da zurzeit der Kampf gegen die Kohleenergie wichtiger sei.

Zurzeit verbraucht Polen jährlich 14,5 Mrd. $\mathrm{m}^{3}$ Gas, in nächster Zukunft aber wird der Bedarf steigen. Dies geht aus den Plänen hervor, Gaskraftwerke zu bauen. Diese Investitionen muss Polen aus verschiedenen Gründen tätigen. Erstens aus ökologischen Gründen. Die polnische Stromproduktion basiert auf Kohle, und das EU-Klimapaket 20-20-20 (bis 2020 sollen der Energieverbrauch und die Treibhausemissionen im Vergleich zu 1990 um 20 Prozent gesenkt werden; der Anteil erneuerbarer Energien soll auf 20 Prozent steigen) stellt harte Forderungen zur Beschränkung der $\mathrm{CO}_{2}$-Emissionen auf: Polen ist verpflichtet, die $\mathrm{CO}_{2}$-Emissionen bis zum Jahr 2020 um 15 Prozent zu reduzieren. Zweitens ist ein großer Teil der Blocks in polnischen Kraftwerken ausgeschöpft und muss in den nächsten Jahren abgeschaltet werden. Gaskraftwerke lassen sich sehr schnell bauen, sie wären also die einzige Chance, die fehlenden Blocks zu ersetzen. Schließlich gibt es noch einen dritten Grund: Die Entwicklung der Windenergie erfordert ergänzende Energiequellen, die eingesetzt werden, wenn der Wind nicht weht. Gaskraftwerke können am schnellsten aktiviert werden und würden sich daher am besten zu diesem Zweck eignen.

\section{Atomkraft als Zeichen von Modernität}

Unter den Mitgliedsländern der Europäischen Union ist Polen am stärksten von Kohle abhängig. 56 Prozent des Bedarfs an Primärenergie wird mit Braunkohle und Steinkohle gedeckt und 90 Prozent des Stroms wird über Kraftwerke und Heizkraftwerke auf der Basis von Kohle erzeugt. Der Steinkohlebergbau ist ein mächtiger Zweig der polnischen Wirtschaft und die Bergleute eine einflussreiche gesellschaftliche Gruppe mit sehr starken Gewerkschaften. Viele Bergwerke arbeiten unrentabel, werden aber aus sozialen Gründen und auch im Hinblick auf die Energiesicherheit gehalten. Die Privatisierung der Bergbaubranche beginnt erst allmählich. Es herrscht die Überzeugung, dass die Kohle, "schwarzes Gold" genannt, eine Versicherungspolice im Bereich Energie ist - und das, obwohl Polen seit einigen Jahren eine negative Bilanz im Außenhandel mit Steinkohle verzeichnet. Die Förderung im Land beträgt 76,2 Mio. t, der Import dagegen beträgt bereits zirka 15 Mio. t. Ein Teil der Kohle kommt aus Russland, was aber keine Emotionen in der öffentlichen Meinung in Hinblick auf die Abhängigkeit vom östlichen Lieferanten hervorruft.

Die Veränderung des polnischen Energiemix ist eine der wichtigsten wirtschaftlichen Herausforderungen des Landes. Die EU betreibt eine Politik, die die Kohle letztendlich ausschließen wird, was in Polen als weite- 
rer Anschlag auf die eigene Energiesicherheit aufgefasst wird. Immer lauter werden die Stimmen, dass Polen aufgrund seiner besonderen Situation um sein Recht auf die Nutzung von Kohle kämpfen sollte. Dennoch sind sich alle der Notwendigkeit bewusst, die Kohlemonokultur zu beschränken. Das Regierungsdokument »Die Energiepolitik Polens bis 2030 « geht davon aus, dass die Bedeutung der Kohle zwar wesentlich ist, sich aber verringern wird. Dies soll durch Verbesserungen der Energieeffizienz bewirkt werden (die heute zweimal niedriger ist als im Durchschnitt der EU), durch die Erhöhung des Anteils von Gas, die Entwicklung erneuerbarer Energien und durch die Atomenergie.

Bisher wurde in Polen keine Atomenergie erzeugt. In der Zeit des kommunistischen Systems war zwar in Żarnowiec (Pommern) mit dem Bau eines Atomkraftwerks begonnen worden, das sich auf die sowjetische Technologie stützte, aber im Jahr 1990 wurden die Arbeiten eingestellt. Zur Idee der Atomenergie kehrte man vor einigen Jahren zurück. Im Jahr 2009 beschloss die Regierung, die Vorbereitungen für den Bau des ersten Atomkraftwerks aufzunehmen. Seine Inbetriebnahme war ursprünglich für 2020 geplant, es zeichnet sich aber schon ab, dass eine so rasche Fertigstellung nicht gelingen wird. Es wurde ein Regierungsbeauftragter für die polnische Atomenergie im Rang des stellvertretenden Wirtschaftsministers berufen. Zu seinen Aufgaben gehören u. a. die Vorbereitung der notwendigen rechtlichen Regulierungen (das Atomrecht), die Berufung der Behörde für die Entwicklung der Atomenergie (Agencja Rozwoju Energetyki Jądrowej) und die Durchführung von Untersuchungen bezüglich der Technologie, der Wahl des Standorts usw. Das Projekt erhält starke politische Unterstützung. In der Atomenergie sehen die Politiker die Chance, Polen von der Kohle zu lösen, die Anforderungen des Umweltschutzes zu erfüllen und Energiesicherheit aufzubauen. Außerdem geht es auch um Modernisierung, um den Wunsch zum Klub der Länder zu gehören, die Atomenergie erzeugen.

Geplant ist, das erste Atomkraftwerk in den Jahren 2016 bis 2022 zu bauen. Bis zum Jahr 2030 sollen bereits zwei Atomkraftwerke mit einer Kapazität von insgesamt 6.000 MW arbeiten. Die Umsetzung dieses Vorhabens liegt bei der Polska Grupa Energetyczna, dem größten polnischen Energiekonzern, der vom Staat kontrolliert wird. Die geschätzten Kosten belaufen sich auf 3-3,5 Mrd. Euro pro 1.000 MW. Die Wahl des Technologielieferanten soll Ende 2014/Anfang 2015 getroffen werden. Das Atomkraftwerk soll in der Technologie der Reaktoren der Generation III bzw. III+ gebaut werden, allerdings wurde noch kein Lieferant für die Installation gewählt.
Der Bau eines Atomkraftwerks weckt heute nur unter Experten und Umweltaktivisten Kontroversen. Zwar begann die Regierung im April eine Informationskampagne in den Medien, um für die Idee des Baus zu werben, doch weckte das keine besonderen Emotionen. Die ganze Angelegenheit ist noch so weit weg, dass sie keine Anti-Atom-Gruppierungen zu wahrnehmbaren Aktionen provoziert. So wird es sicherlich bleiben, bis das Projekt eine deutlichere Gestalt annimmt. Bisher wurde noch nicht einmal ein Standort für das erste Atomkraftwerk gewählt. Diese Zurückhaltung in den Reaktionen auf das Atomprogramm ergibt sich aus der Tatsache, dass es in letzter Zeit zu einer ganzen Flut von unterschiedlichen Projekten im Bereich Energie kam. Immer mehr Gesellschaften informieren über ihre Absicht, unterschiedliche Kraftwerke zu bauen - Kohle-, Atom-, Gas-, Windkraftwerke usw. - aber nicht alle Projekte sind realistisch und nicht bei allen ist der Wille erkennbar, dass sie realisiert werden sollen.

\section{Welche Investitionen sind sinnvoll?}

Die polnischen Kraftwerke decken zurzeit den Bedarf an Strom, der im Spitzenwert 24.000-25.000 MW erreicht. Die Gesamtkapazität aus den unterschiedlichen Quellen beträgt 35.000 MW. Theoretisch gibt es kein Problem eines Kapazitätsdefizits. Praktisch gesehen entstand jedoch die Mehrheit der Blocks in den Kraftwerken vor 1989 und erfordert immer häufigere Reparaturen. In allernächster Zeit müssen sie außer Betrieb genommen werden. Der Bau neuer Blocks in den bereits bestehenden Kraftwerken geht sehr langsam voran. Die einzige große Investition in der letzten Zeit war das Kraftwerk Bełchatów II mit einer Kapazität von 858 MW. Der Brennstoff ist hier Braunkohle. Das Problem, die Kapazität in den Braunkohlekraftwerken auszubauen, verbindet sich mit dem Problem, die Emissionsrechte für $\mathrm{CO}_{2}$ im Rahmen des EU-Emissionshandels (ETS) zu erwerben. Es ist absehbar, dass dies eine deutliche Preissteigerung für Strom mit sich bringen wird, wobei noch nicht klar ist, in welcher Höhe, denn es wurde noch nicht über die kostenlosen Emissionsberechtigungen entschieden, die die polnischen Kraftwerke in den Jahren 2013 bis 2019 erhalten sollen.

Polen war bislang das Land, das viel energieintensive Industrie aufweist, u. a. wegen der niedrigen Energiepreise. Dies ändert sich jetzt, was zur Folge hat, dass die energieintensive Industrie aus Polen abwandert; dieser Prozess hat bereits begonnen. Hervorzuheben ist, dass der Energieverbrauch des Bruttoinlandsprodukts (BIP) fällt. Zwischen den Jahren 2000 und 2008 stieg das BIP Polens um 38,6 Prozent und der Verbrauch der Primärenergie nur um 6,8 Prozent. Den Investoren fällt es schwer, den realen Energiebedarf der polnischen Wirt- 
schaft einzuschätzen und in diesem Zusammenhang, welche Investitionen sinnvoll wären. Diskutiert wird über die Zukunft der Technologie der $\mathrm{CO}_{2}$-Abscheidung und Speicherung (carbon capture and storage-CCS). Die erste Anlage dieser Art sollte im Kraftwerk Bełchatów entstehen, aber es ist noch unklar, ob sich geologisch geeignete Standorte finden lassen, wo $\mathrm{CO}_{2}$ eingelagert werden kann, so dass der Eigentümer des Kraftwerks die Investition in CCS als zu kostspielig erachtet. Neben der CCS-Technologie, die eine Möglichkeit sein könnte, die Nutzung von Kohle mit der Klimapolitik in Einklang zu bringen, wird auch die Möglichkeit der Vergasung von Kohle in Betracht gezogen.

Die Entwicklung der Energie aus erneuerbaren Quellen trifft auf größere Probleme. Dies hängt vor allem mit den hohen Investitionskosten und der nicht entsprechend ausgestatteten Infrastruktur zusammen. Vor allem betrifft dies den Anschluss von Windparks an das Netz sowie den Aufbau von Produzent=Konsument Energieangeboten, das heißt solchen, bei der ein Teil der individuellen Energieabnehmer unter Einsatz von kleinen Windgeneratoren, Fotovoltaik usw. auch Energieerzeuger ist.

Ein großer Teil der Verpflichtungen im Bereich der erneuerbaren Energie wird über die Verbrennung von Biomasse eingelöst. Im Kraftwerk Połaniec, das zu GdF Suez gehört, entstand vor kurzer Zeit der weltweit größte Block zur Verbrennung von Biomasse. Er hat eine Kapazität von 190 MW. Doch wird im größten Teil der Kraftwerke Biomasse in herkömmlichen Kohleblocks gleichzeitig mit Kohle und Holz verbrannt. Die Abholzung von Wäldern und ihre Verbrennung zwecks Gewinnung grüner Energie riefen Proteste hervor. Nach einer Welle der Kritik wird Holz den Status von Biomasse verlieren. Auf diese Weise aber hatte die Energiewirtschaft versucht, den eingeforderten Anteil an Energie aus erneuerbaren Quellen zu stellen.

Eine zweite Quelle für erneuerbare Energie sind Wasserkraftwerke, eine dritte Quelle Wind. Die Kapazität der gegenwärtig arbeitenden Windparks beträgt 1.600 MW. Sonnenenergie oder Erdwärme wird praktisch nicht genutzt. Die weitere Entwicklung der erneuerbaren Energien wird vom neuen Unterstützungssystem abhängen, denn das aktuelle endet im Jahr 2017. Zurzeit wird an einem neuen Gesetz zu erneuerbaren Energien gearbeitet.

\section{Die aktuellen Herausforderungen}

Polen steht heute vor einigen großen Herausforderungen im Bereich Energiepolitik. Erstens muss das traditionelle Energiemodell, das auf fossilen Brennstoffen basiert, geändert werden. Zweitens müssen enorme Investitionen getätigt werden, um diejenigen Kraftwerke zu ersetzen, die abgeschaltet werden müssen, um die Netze der Strom- und Gaspipelines zu modernisieren und um die Gewinnung erneuerbarer Energie auszubauen. Drittens muss die Energieinfrastruktur im Land mit der der EU integriert werden. Dies erfordert einen Ausbau der grenzüberschreitenden Verbindungen, sowohl im Bereich Strom als auch im Bereich von Gasund Ölpipelines. Viertens muss der Energiemarkt liberalisiert werden. Zurzeit wird der Erdgasmarkt vollständig reguliert. Die Preistarife für Gas legt die Behörde für Regulierung der Energiewirtschaft (Urząd Regulacji Energetyki) fest, was dazu führen kann, dass die Europäische Kommission Schritte gegen Polen einleitet. Planmäßig ist eine Liberalisierung des Erdgasmarktes ab 2013 vorgesehen. Auch die Strompreise für den individuellen Verbraucher werden reguliert. Fünftens müssen Gesetze geändert werden. Zurzeit wird ein Gesetzespaket erarbeitet, das die Bedingungen für die notwendigen Reformen schaffen soll. Dabei handelt es sich um das Energierecht, das Gasrecht und das Gesetz über erneuerbare Energien. Im vergangenen Jahr wurde bereits das Gesetz über Energieeffizienz verabschiedet.

Übersetzung aus dem Polnischen: Silke Plate

Über den Autor

Adam Grzeszak ist Publizist der Wochenzeitung POLITYKA, Fachgebiet Wirtschaftsfragen.

Das im Artikel genannte Regierungsdokument »Die Energiepolitik Polens bis 2030« ist auf Englisch (»Energy Policy of Poland until 2030«) abrufbar unter: http://www.mg.gov.pl/files/upload/8134/Polityka\%20energetyczna\%20ost_en.pdf (abgerufen am 15.05.2012). 


\section{Der polnische Energiemix}

Grafik 1: Der Bedarf an Primärenergie nach Energiequellen

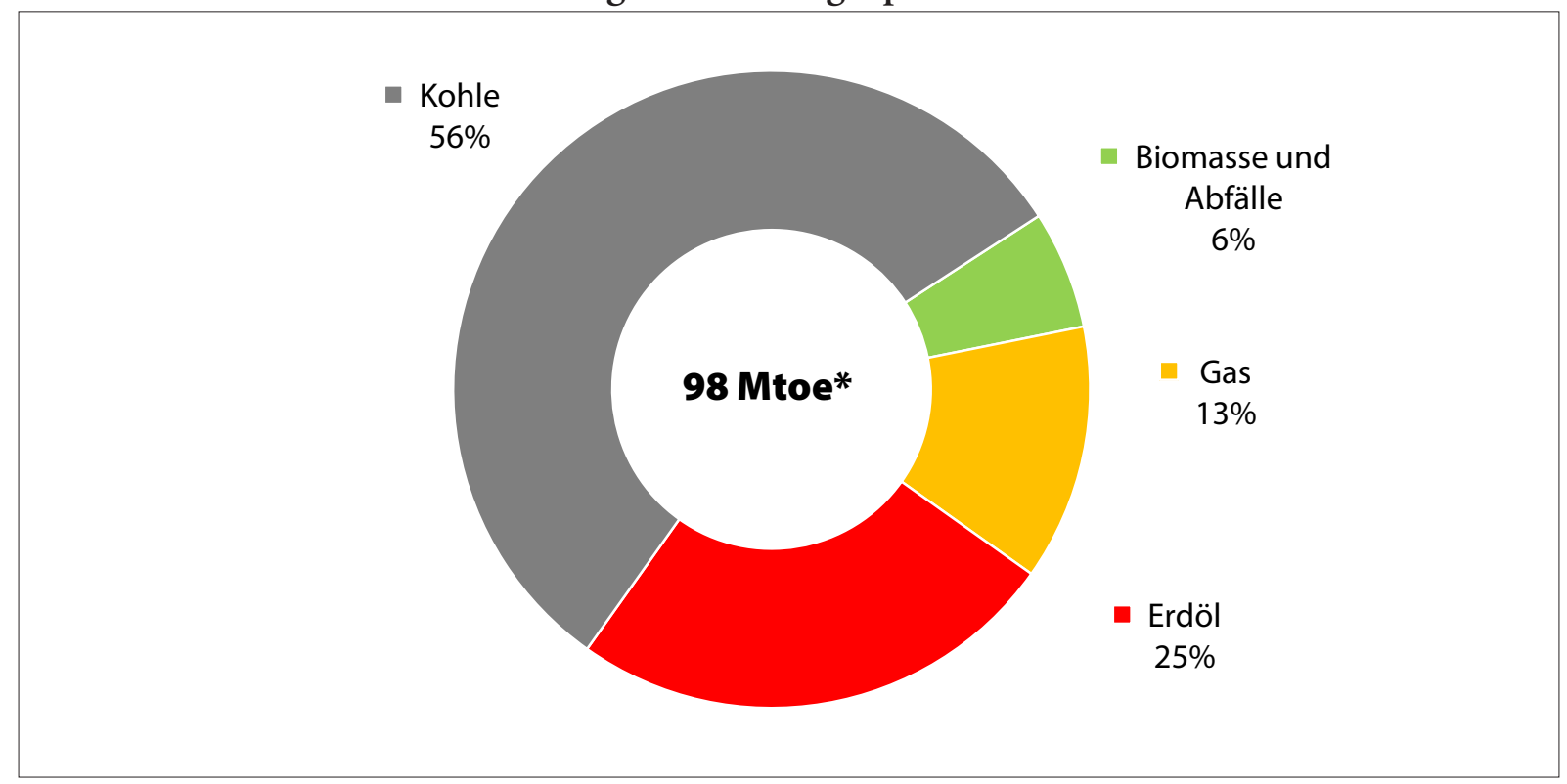

* Megatonne Öleinheiten

Quellen: Agencja Rynku Energii (2011) und IEA International Energy Agency (2010)

Grafik 2: Der Endenergieverbrauch nach Energieträgern

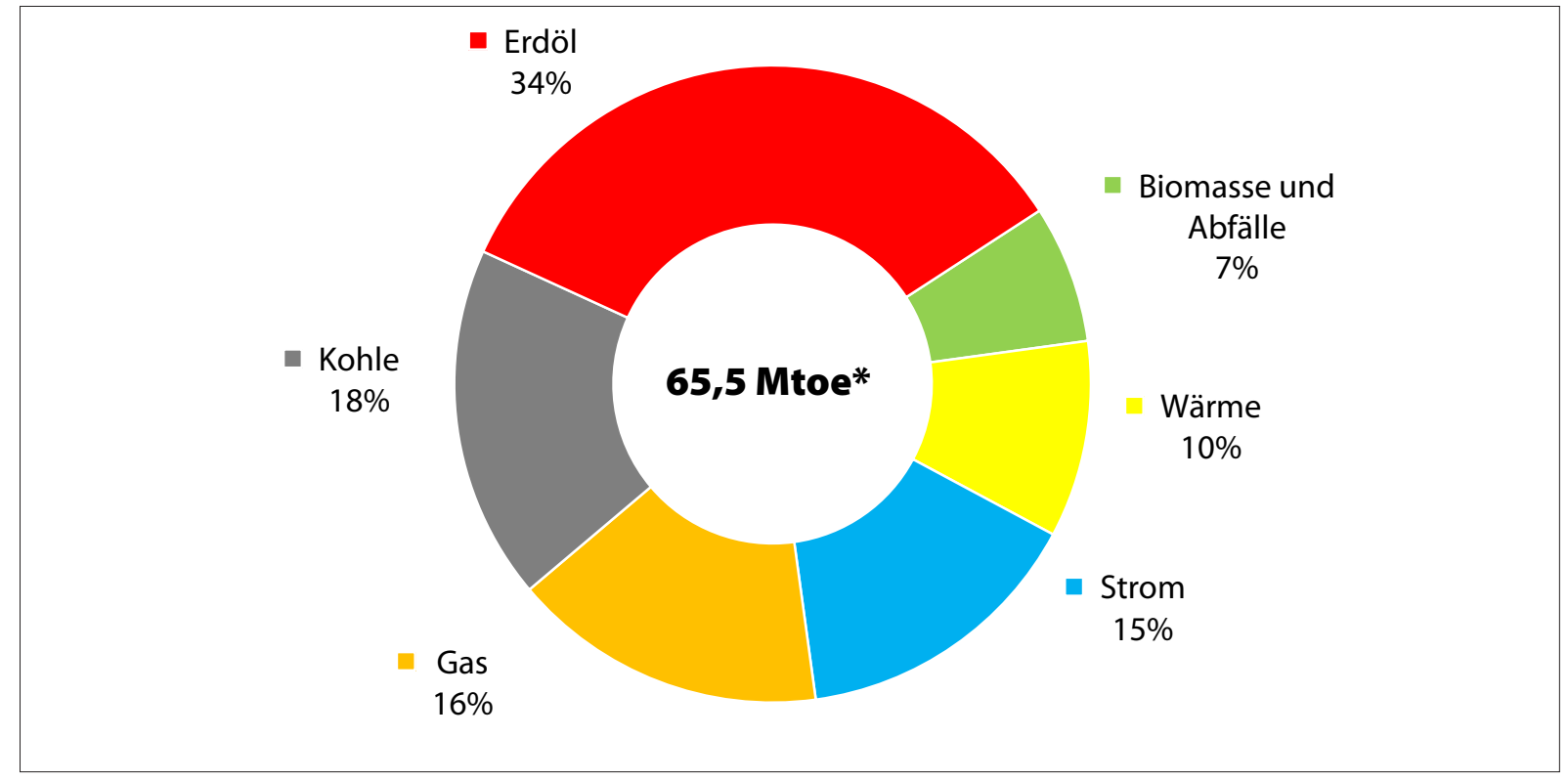

* Megatonne Öleinheiten

Quellen: Agencja Rynku Energii (2011) und IEA International Energy Agency (2010) 
Grafik 3. Stromerzeugung nach Technologien

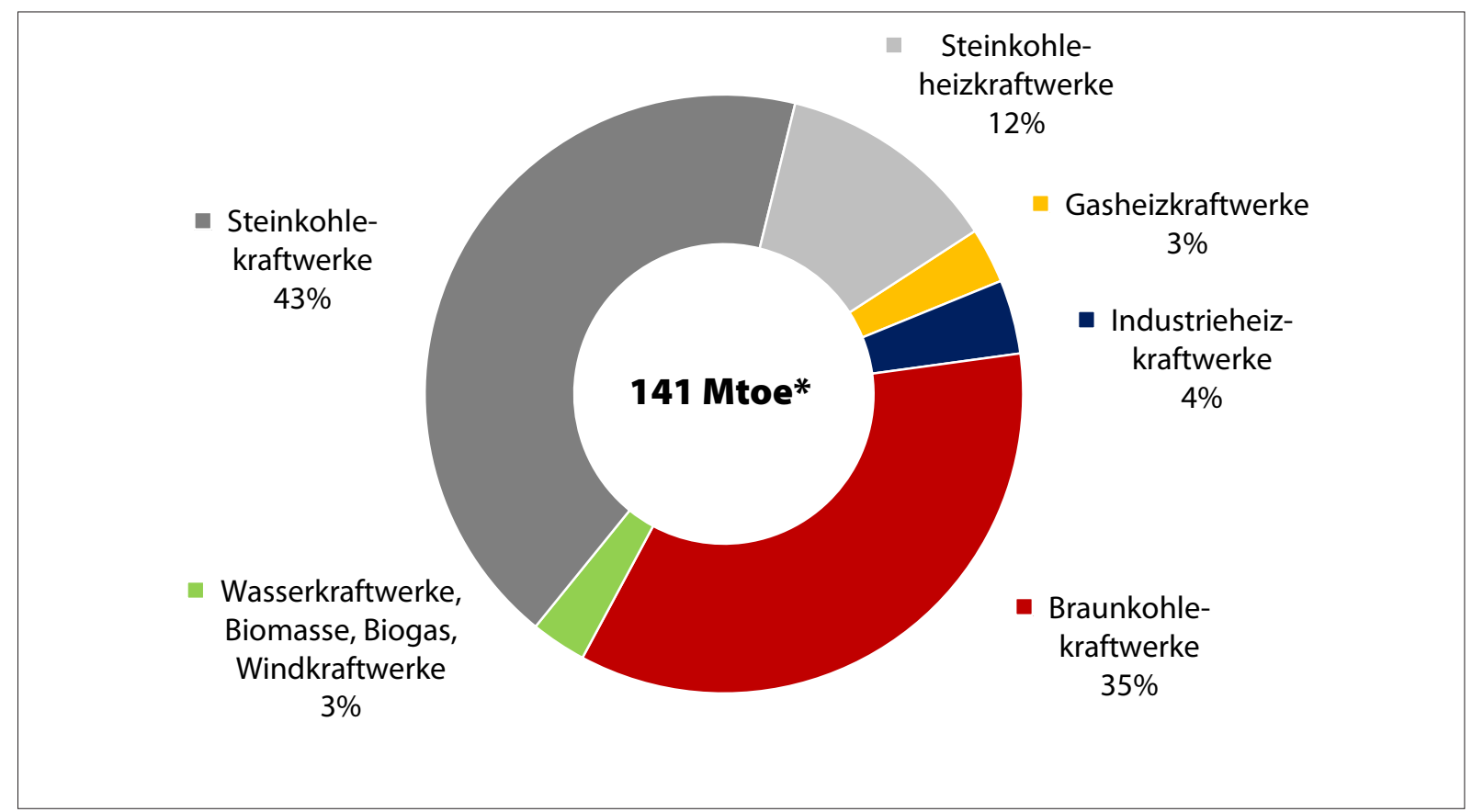

* Megatonne Öleinheiten

Quellen: Agencja Rynku Energii (2011) und IEA International Energy Agency (2010)

Grafik 4. Nettokapazität der Kraftwerke und Technologien zur Stromerzeugung

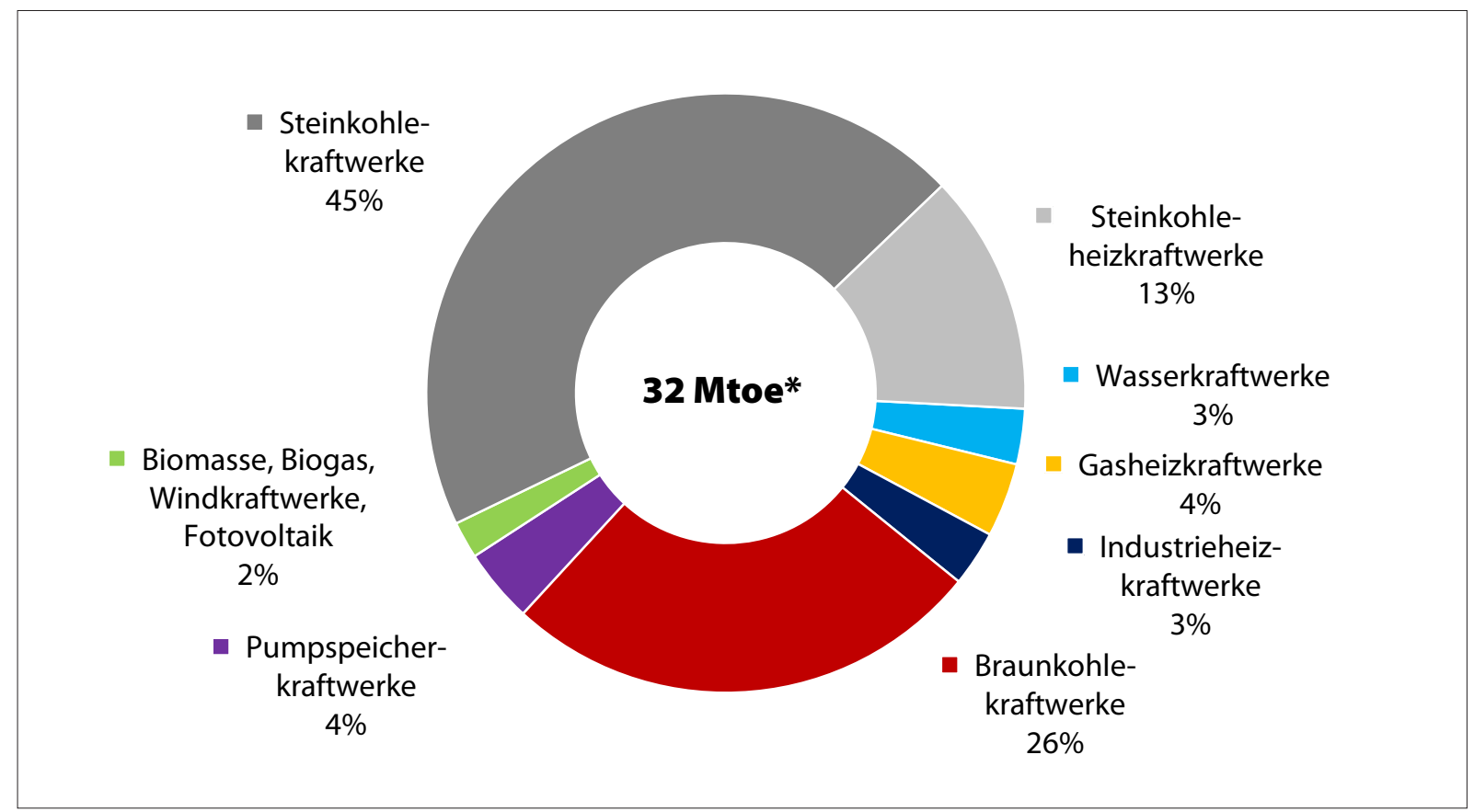

* Megatonne Öleinheiten

Quellen: Agencja Rynku Energii (2011) und IEA International Energy Agency (2010) 


\section{Vom 1. bis zum 14. Mai 2012}

\begin{tabular}{|c|c|}
\hline 01.05 .2012 & $\begin{array}{l}\text { Auf dem Parteitag der Palikot-Bewegung (Ruch Palikota) in Warschau stellt der Parteivorsitzende Janusz } \\
\text { Palikot das Projekt »Korrektur des Kapitalismus« vor, das eine Reihe von gesellschaftspolitischen und wirt- } \\
\text { schaftlichen Gesetzesentwürfen umfasst. Angestrebtes Ziel sei u. a. die Vollbeschäftigung in Polen. }\end{array}$ \\
\hline 02.05 .2012 & $\begin{array}{l}\text { In vielen Städten beginnt die Unterschriftensammlung für einen sogenannten Bürgergesetzesentwurf, der } \\
\text { sich gegen die Reform des Geschichtsunterrichts in der Oberschule (liceum) richtet. Diese wird im Septem- } \\
\text { ber in Kraft treten. Im Vorfeld hatten bereits Hungerstreiks gegen die Reform stattgefunden. }\end{array}$ \\
\hline 03.05 .2012 & $\begin{array}{l}\text { In seiner Rede zum Staatsfeiertag des 3. Mai, dem Tag der Verfassung von 1791, thematisiert Staatspräsident } \\
\text { Bronisław Komorowski die Verantwortung aller Bürger für Polen. Dazu gehöre auch, sprachliche Übertrei- } \\
\text { bungen und Verunglimpfungen zur Charakterisierung der Lage im Land zu unterlassen, die nur den par- } \\
\text { teispezifischen Interessen dienen würden. }\end{array}$ \\
\hline 03.05 .2012 & $\begin{array}{l}\text { Ministerpräsident Donald Tusk zeigt sich überzeugt, dass die ukrainische Regierung den Konflikt, der um } \\
\text { die Haftbedingungen für die ehemalige ukrainische Ministerpräsidentin Julia Timoschenko international } \\
\text { entbrannt ist, auf humanitäre Weise lösen wird. Tusk spricht sich gegen den Appell des Vorsitzenden von } \\
\text { Recht und Gerechtigkeit (Prawo i Sprawiedliwość-PiS), Jarosław Kaczyński, aus, der Ukraine die Ausrich- } \\
\text { tung der Fußballeuropameisterschaft EURO 2012, die im Juni und Juli in Polen und der Ukraine stattfin- } \\
\text { den soll, zu entziehen. Tusk fordert dazu auf, Briefe an die ukrainische Regierung zu schreiben und zu for- } \\
\text { dern, dass Timoschenko zur EURO } 2012 \text { freigelassen wird. }\end{array}$ \\
\hline 04.05 .2012 & $\begin{array}{l}\text { In Litoměřice (Tschechien) beschließen die Verteidigungsminister der Visegrád-Gruppe (Polen, Tschechien, } \\
\text { Slowakei und Ungarn) den Aufbau einer gemeinsamen Militäreinheit in der Europäischen Union, die 3.000 } \\
\text { Soldaten zählen wird. Verteidigungsminister Tomasz Siemoniak teilt mit, dass an der Spitze der Militär- } \\
\text { einheit ein polnischer Offizier stehen wird und } 1.200 \text { polnische Soldaten zu der Einheit gehören werden. }\end{array}$ \\
\hline 06.05 .2012 & $\begin{array}{l}\text { Der Vorsitzende von Solidarisches Polen von Zbigniew Ziobro (Solidarna Polska Zbigniewa Ziobra), Zbigniew } \\
\text { Ziobro, fordert von Ministerpräsident Donald Tusk eine konsequente Haltung gegenüber dem Boykott der- } \\
\text { jenigen Spiele der Fußballeuropameisterschaft EURO 2012, die in der Ukraine ausgetragen werden sollen. } \\
\text { Einerseits lehne Tusk den Boykottaufruf von Jarosław Kaczyński (Recht und Gerechtigkeit/Prawo i Spra- } \\
\text { wiedliwość - PiS) ab, andererseits nehme er nicht Stellung, wenn seine Parteikollegen in der Europäischen } \\
\text { Union den Boykott unterstützen. Ziobro unterstreicht, dass ein Boykott eine Annäherung der Ukraine an } \\
\text { Russland befördern könne. }\end{array}$ \\
\hline 07.05.2012 & $\begin{array}{l}\text { Die Gesellschaft der Allgemeinmediziner (Stowarzyszenie Lekarzy Praktyków) teilt dem Zentralen Anti- } \\
\text { korruptionsbüro (Centralne Biuro Antykorupcyjne - CBA) einen weiteren Korruptionsverdacht gegenüber } \\
\text { dem Gesundheitsministerium im Zusammenhang mit dem seit Jahresanfang geltenden Arzneimittelgesetz } \\
\text { und der Festlegung von Arzneimittelpreisen mit. }\end{array}$ \\
\hline 08.05 .2012 & $\begin{array}{l}\text { Auf einer Pressekonferenz teilt Regierungssprecher Paweł Graś mit, dass } 80 \text { Prozent der polnischen Infra- } \\
\text { strukturprojekte für die Fußballeuropameisterschaft EURO 2012, die im Juni und Juli in Polen und der } \\
\text { Ukraine stattfinden wird, bereits fertig gestellt worden sind. Außerdem gibt er bekannt, dass die polnische } \\
\text { Regierung keinen Einfluss auf die Gästeliste für die Spiele in Polen nehmen wird. Hintergrund sind die } \\
\text { Haftbedingungen der inhaftierten ehemaligen ukrainischen Ministerpräsidentin Julia Timoschenko in der } \\
\text { Ukraine, was zu Boykottaufrufen und -ankündigungen geführt hatte. }\end{array}$ \\
\hline 09.05.2012 & $\begin{array}{l}\text { Staatspräsident Bronisław Komorowski appelliert an die ukrainische Regierung, die anachronistische } \\
\text { Gesetzgebung aufzuheben, die es erlaubt, für politische Entscheidungen Haftstrafen zu verhängen. Hin- } \\
\text { tergrund ist die Inhaftierung und der erneute Prozess gegen die ehemalige ukrainische Ministerpräsidentin } \\
\text { Julia Timoschenko. Polen werde weiterhin die europäischen Ambitionen der Ukraine unterstützen. Außer- } \\
\text { dem sprach sich Komorowski gegen einen Boykott der Ukraine und der Fußballeuropameisterschaft EURO } \\
2012 \text { in der Ukraine aus, die im Sommer gemeinsam in Polen und der Ukraine ausgeführt werden soll. }\end{array}$ \\
\hline 10.05 .2012 & $\begin{array}{l}\text { Der Vorsitzende der Demokratischen Linksallianz (Sojusz Lewicy Demokratycznej - SLD), Leszek Miller, } \\
\text { appelliert an Ministerpräsident Donald Tusk, das Gesetzgebungsverfahren zur Rentenreform zu stoppen. } \\
\text { Einer der Gründe sei, dass die Regierung keinen Willen gezeigt habe, sich um eine breitere Unterstützung } \\
\text { der Reform von Seiten der Opposition zu bemühen. }\end{array}$ \\
\hline 11.05 .2012 & $\begin{array}{l}\text { Nach einer hitzigen Debatte wird im Sejm über die Rentenreform abgestimmt. Für die schrittweise Anhe- } \\
\text { bung und Angleichung des Renteneintrittsalters auf } 67 \text { Jahre für Männer und Frauen stimmen } 268 \text { Abge- } \\
\text { ordnete, } 185 \text { stimmen dagegen, zwei Abgeordnete enthalten sich. Die Debatte wurde von mehrtägigen } \\
\text { Demonstrationen vor dem Parlamentsgebäude begleitet. }\end{array}$ \\
\hline
\end{tabular}




\begin{tabular}{|l|l|}
\hline 12.05 .2012 & $\begin{array}{l}\text { Der Abgeordnete von Recht und Gerechtigkeit (Prawo i Sprawiedliwość - PiS), Tomasz Górski, tritt zur } \\
\text { Fraktion Solidarisches Polen von Zbigniew Ziobro (Solidarna Polska Zbigniewa Ziobra) über. Dies sei eine } \\
\text { dynamische Gruppierung des Mitte-Rechts-Spektrums, in der er sich für den polnischen Mittelstand enga- } \\
\text { gieren wolle, so Górski. }\end{array}$ \\
\hline 14.05 .2012 & $\begin{array}{l}\text { Nach jüngsten Angaben des Ministeriums für regionale Entwicklung sind zwei Drittel der finanziellen Mit- } \\
\text { tel des Programms »Entwicklung Ostpolens« bereits abgerufen worden. Die Gesamtsumme von zirka 10 } \\
\text { Mrd. Zloty für die Jahre 2007 bis 2013 setzt sich aus Mitteln der Europäischen Union und des öffentlichen } \\
\text { Haushalts Polens zusammen und fördert Infrastrukturprojekte in den Woiwodschaften Ermland-Masuren } \\
\text { (województwo warmińsko-mazurskie), Podlachien (podlaskie), Lublin (lubelskie), Vorkarpaten (podkar- } \\
\text { packie) und Heiligkreuz (świętokrzyskie). }\end{array}$ \\
\hline
\end{tabular}

Sie können die gesamte Chronik seit 2007 auch auf http://www.laender-analysen.de/polen/ unter dem Link "Chronik« lesen. 


\section{Deutsch-Polnische} Sommerakademie

\section{2. bis 9. September 2012}

\section{Darmstadt, Deutsches Polen-Institut}

Die Interdisziplinäre Deutsch-Polnische Sommerakademie gibt Nachwuchswissenschaftlerinnen und -wissenschaftlern die Gelegenheit, ihre aktuellen Forschungsprojekte in einem multidisziplinären Kreis vorzustellen und über Aspekte von Geschichte und Gegenwart Polens, Deutschlands und der deutschpolnischen Beziehungen in einem europäischen Kontext zu diskutieren. Als Dozentinnen konnten für die Sommerakademie 2012 die Historikerin Claudia Kraft (Siegen) und die Literaturwissenschaftlerin Magdalena Marszałek (Potsdam) gewonnen werden. Auch die Schriftstellerin Olga Tokarczuk nimmt an der Sommerakademie teil.

Seminarblöcke, Vorträge, kulturelle Veranstaltungen und eine Exkursion ergänzen das Programm; es besteht die Möglichkeit zur Arbeit in Bibliothek und Archiven des Deutschen Polen-Instituts. Ziel ist es, junge Wissenschaftlerinnen und Wissenschaftler aus beiden Ländern auf unkonventionelle Weise miteinander zu vernetzen und für die Komplexität des deutsch-polnischen Beziehungsgeflechts zu sensibilisieren.

Angesprochen sind Doktorandinnen und Doktoranden, Postdocs, aber auch fortgeschrittene Studierende kurz vor dem Abschluss, vornehmlich aus Deutschland und Polen, die sich in ihrer aktuellen wissenschaftlichen Arbeit mit Deutschland, Polen bzw. den deutsch-polnischen Beziehungen beschäftigen. Voraussetzung der Teilnahme ist eine halbstündige Projektvorstellung. Arbeitssprache ist Deutsch, nach Absprache auch Polnisch oder Englisch. Teilnehmerbeitrag: 100 Euro für deutsche / 60 Euro für polnische Teilnehmer. Kosten für Übernachtung, Verpflegung und Reisekosten werden vom Veranstalter übernommen.

Bewerbungsschluss ist der 1. Juni 2012.

Weitere Informationen und Anmeldeformular: www.sommerschule-polen.de

Kontakt: Manfred Mack, mack@dpi-da.de

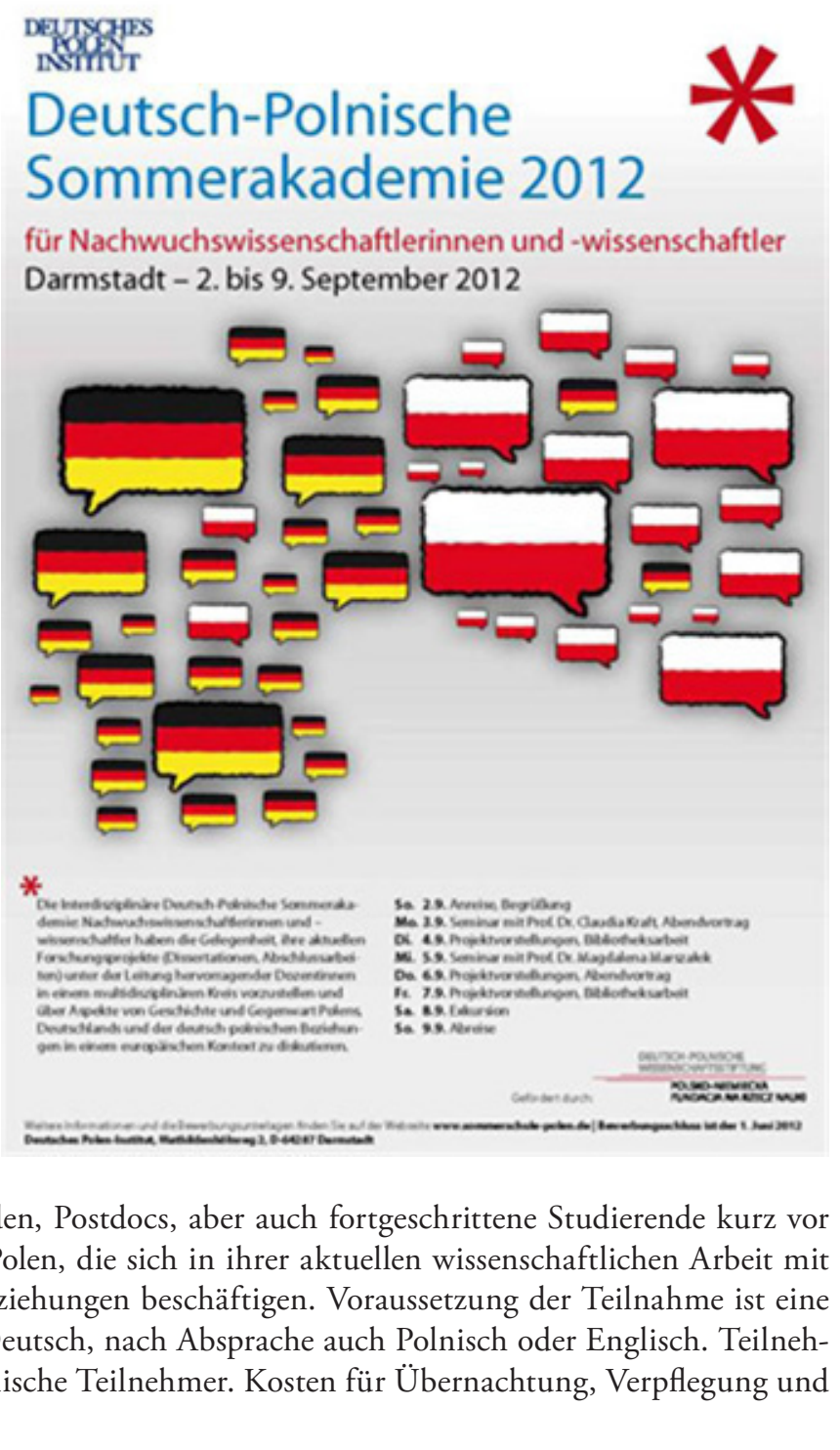

für Nachwuchswissenschaftlerinnen und -wissenschaftler Darmstadt - 2. bis 9. September 2012

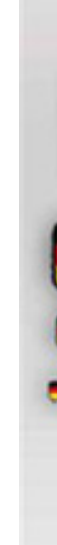


Die Polen-Analysen erscheinen zweimal monatlich als E-Mail-Dienst. Sie werden gemeinsam vom Deutschen PolenInstitut Darmstadt, der Bremer Forschungsstelle Osteuropa und der Deutschen Gesellschaft für Osteuropakunde herausgegeben.

Ein Archiv der Polen-Analysen finden Sie im Internet unter www.laender-analysen.de/polen

Kostenloses Abonnement unter http://www.deutsches-polen-institut.de/Newsletter/subscribe.php

Diese Analysen finden Sie online als Lizenzausgabe auf

bpb.de

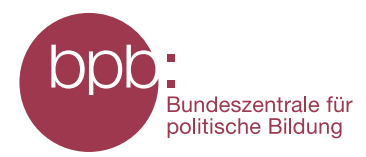

\section{Deutsches Polen-Institut Darmstadt}

Das Deutsche Polen-Institut Darmstadt (DPI) ist ein Forschungs-, Informations-, und Veranstaltungszentrum für polnische Kultur, Geschichte, Politik, Gesellschaft und die deutsch-polnischen Beziehungen, die sich im Kontext der europäischen Integration entwickeln. Das seit März 1980 aktive und bis 1997 von Gründungsdirektor Karl Dedecius geleitete Institut ist eine Gemeinschaftsgründung der Stadt Darmstadt, der Länder Hessen und Rheinland-Pfalz sowie des Bundes. 1987 wurden die Kultusminister der Länder und 2011 das Auswärtige Amt weitere institutionelle Träger. Einen wesentlichen Beitrag zur Verwirklichung der Institutsziele leisten private Stiftungen. Das DPI hat satzungsgemäß die Aufgabe, durch seine Arbeit zur Vertiefung der gegenseitigen Kenntnisse des kulturellen, geistigen und gesellschaftlichen Lebens von Polen und Deutschen beizutragen.

Ziel der Vermittlertätigkeit des DPI ist es, »die zu interessieren, auf die es politisch, wirtschaftlich, gesellschaftlich und kulturell im deutsch-polnischen Verhältnis ankommt (Leitlinien 1997). Es geht um die Entscheider und Multiplikatoren in Politik, Kultur, Bildung, Verwaltung, Medien und Wirtschaft und, wesentlich stärker ausgeprägt als bisher, um das Hineinwirken in Wissenschaft, Forschung und Bildung.

Derzeit bemüht sich das DPI in Kooperation mit den verstreuten Orten wissenschaftlicher Polen-Kompetenz an deutschen Hochschulen und Forschungsinstituten verstärkt darum, ausgehend von einer Bestandsaufnahme deutscher Polen-Forschung Ort wissenschaftlicher Forschung und verbindendes, vernetzendes und kooperierendes Zentrum zu werden. Ausgangspunkt der Neuausrichtung ist die kaum mehr kontrollierbare Dynamik des Rückbaus der Ressourcen der wissenschaftlichen Polen-Kompetenz in den unterschiedlichen Disziplinen. Mit der über 60.000 Bände zählenden multidisziplinären Fachbibliothek für Polen, die eine einzigartige Sammlung polnischer Literatur in der Originalsprache und in deutscher Übersetzung umfasst, ist das DPI bereits ein geschätzter Ort der Recherche und des wissenschaftlichen Arbeitens. (www.deutsches-polen-institut.de)

\section{Forschungsstelle Osteuropa an der Universität Bremen (www.forschungsstelle.uni-bremen.de)}

1982 gegründet, widmet sich die Forschungsstelle Osteuropa an der Universität Bremen der interdisziplinären Analyse der Länder Ost- und Ostmitteleuropas in Zeitgeschichte und Gegenwart. Der Forschungsschwerpunkt liegt dabei auf der Rolle von "Dissens und Konsens«, von Opposition und Zivilgesellschaft in ihrem historischen, politischen, gesellschaftlichen und kulturellen Kontext. Die Forschungsstelle besitzt in ihrem Archiv eine einzigartige Sammlung alternativer Kulturgüter und unabhängiger Texte aus den ehemaligen sozialistischen Ländern. Darunter befindet sich auch eine umfangreiche Sammlung des "Zweiten Umlaufs», die das Schrifttum und Dokumente unabhängiger Initiativen und gesellschaftlicher Gruppen in Polen aus der Zeit von 1976 bis zum Umbruch umfasst. Hinzu kommt eine umfangreiche Bibliothek mit wissenschaftlicher Literatur. Mit Archiv, Bibliothek und zwei wissenschaftlichen Abteilungen ist die Forschungsstelle auch eine Anlaufstelle sowohl für Gastwissenschaftler als auch für die interessierte Öffentlichkeit.

Eine der Hauptaufgaben der Forschungsstelle ist die Information der interessierten Öffentlichkeit. Dazu gehören unter anderem regelmäßige E-Mail-Informationsdienste für Politik, Wirtschaft, Zivilgesellschaft und Medien. 